\title{
Proposal of a Cloud Computing System for Management of Health Data
}

\author{
Chandeep Singh \\ Sachdev \\ B.E Student, SAKEC, \\ Chembur, Department of \\ Information Technology, \\ Mumbai University, India \\ Altamash Shaikh \\ B.E Student, SAKEC, \\ Chembur, Department of \\ Information Technology, \\ Mumbai University, India
}

\author{
Maha Singh Matta \\ B.E Student, SAKEC, \\ Chembur, Department of \\ Information Technology, \\ Mumbai University, India
}

\author{
Manpreet Singh Kheberi \\ B.E Student, SAKEC, \\ Chembur, Department of \\ Information Technology, \\ Mumbai University, India
}

\author{
Theres Bemila \\ Assistant Professor, SAKEC, \\ Chembur, Department of \\ Information Technology, \\ Mumbai University, India
}

\author{
Dhanashree K. \\ Toradmalle \\ Assistant Professor, SAKEC, \\ Chembur, Department of \\ Information Technology, \\ Mumbai University, India
}

\begin{abstract}
The paper proposes a Cloud based model for the management of Health data of Patients. The records of the Patient will be centralized by storing them in a Cloud, where in the time of need the authorized Doctors will access the medical records of the Patients. The Patient's medical record includes reports of the patient such as $\mathrm{x}$ ray report, blood report, etc along with the prescription given by the various Doctors. The proposed model is designed to replace the existing medical record management system where the records are stored on a hard copy. The scalability and security are an important issue for health record management and hence are present in the proposed model of Cloud based health record management System known as Cloud based Health First which is built according to the relevant standards.
\end{abstract}

\section{General Terms}

Cloud, Doctor, Patient

\section{Keywords}

Health First, Cloud Computing, Openshift, Records

\section{INTRODUCTION}

Cloud based Health First is a Cloud data storage system where medical data of Patients are centralized. Medical records form an important part of the management of a Patient. Patient data include medical records of the patient that can be used for referral, as and when needed. It is important for Doctors and medical organizations to properly maintain the records of Patients so that patient records can be analyzed and better conduct can be planned accordingly. Also, the records will be a help in eliminating issues of medical carelessness.

The legal system relies mainly on documentary evidence in a situation where medical carelessness is assumed by the Patient or his/her relatives. In an accusation of negligence, this is very often the most important evidence deciding on the sentencing or acquittal of the Doctor. With the increasing use of medical insurance for treatment, the insurance companies also require proper record keeping to prove the Patient's demand for medical operating cost. Improper record keeping can result in rejection of medical claims. Medical records include a variety of documentation of Patient's history, clinical findings, diagnostic test results, preoperative care, operation notes, post-operative care, and daily notes of a Patient's progress, blood bank and medications. There should be proper recording of nursing care, laboratory data, reports of diagnostic evaluations, pharmacy records, and billing processes. This means that the paramedical and nursing staff also should be trained in proper protection of Patient records. Medical record keeping is a specialized area in bigger training and corporate hospitals with separate medical records officers managing these issues. [1]

Digitalized record system stores health information basically falls into two categories: Cloud-based or Client-server. In Cloud-based system, a medical practitioner's data is stored on external servers and can be accessed via the web, requiring only a computer with an Internet connection. Client-server systems store data in-house, requiring a server, hardware and software be installed in the physician's office. [2]

In future, medical clinics and hospitals will be forced to adopt data digitization \& centralization over the next few years because customer demands will be a far more powerful mandate than governmental mandates can ever be. The healthcare industry will also be encouraged to begin adopting the new technology as the cost savings and positive impact on Patients become well understood by the industry [3]

The several medical reasons that encouraged us to develop this system is described in the next section.

\section{MOTIVATION}

Traditional method for storing medical records includes paper work such as prescription, books and various other reports. This method has several limitations at certain point and it is tedious. India is yet to embrace IT in every needed sector and hence still relies on paper to store for health care systems. Hence there is a need for systematic storage of medical data and history, in detail, of an individual from birth to death. Consider when someone visit a Doctor he/she may ask for the Patient previous medical records. The Doctor may expect the Patients to have all their history by heart or have all the necessary documents pertaining to their medical records with them. Many of the times it becomes difficult for the Patient to maintain these records or to by heart them. What if there is a system available that manages all the details and stores the details from prescription, operating details, patient history, physical examination, treatment plans, consent forms and other medical records at your fingertips? No need for the 
Patient to maintain his/her medical records. In this paper the proposed model of Cloud data storage system helps to store all the Patient details regarding health, which will help the Patient as well as the Doctor to easily store and access his/her records and it can make Patients life hassle free and makes the Doctor task of diagnoses much easier. To overcome such problems, this paper proposes a method that is described in the subsequent section.

\section{ARCHITECTURE}

Cloud based Health First is a Cloud data storage System which handles medical records of the people. The purpose of this service is to digitize \& centralized medical records. This service will implement an effective web application for medical records. The Figure1 present the architecture of Cloud based Health First System which includes the components namely cloud clients, Openshift cloud, Cloud Server and database.

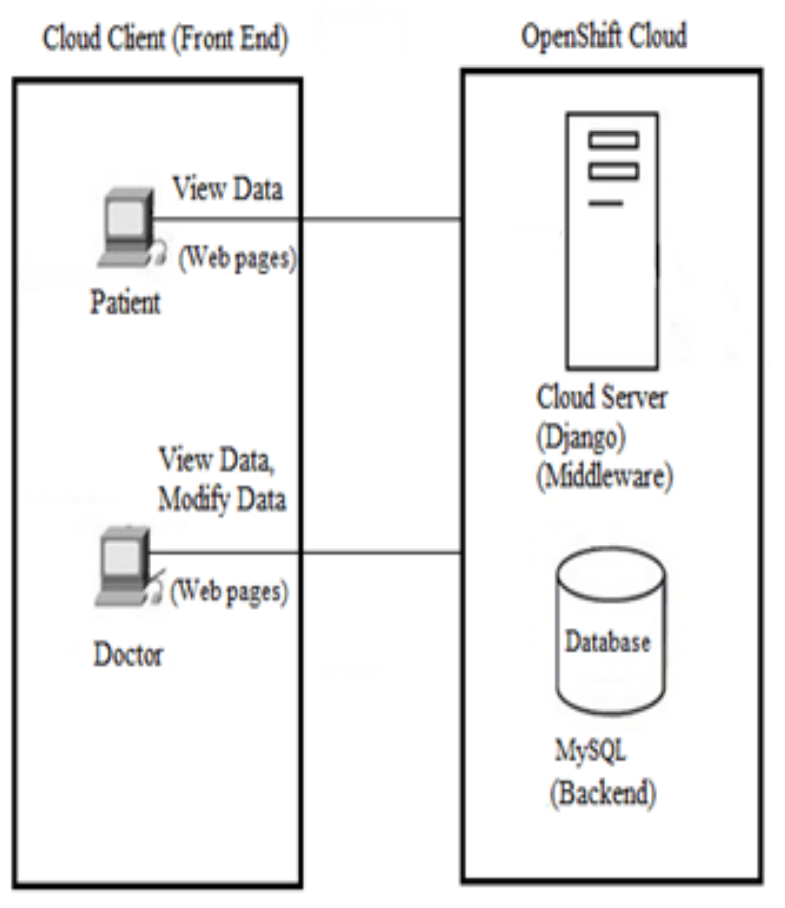

Fig 1: Architecture of Cloud Based Health First

- Cloud Clients: The Cloud data storage System provides the GUI necessary for understanding and operating on the data. This webpage is generated in HTML5 and CSS3. The complete system interaction is managed with Django framework. The Openshift Cloud system interacts with the web pages and provides the necessary data from the Database.

- Openshift: Openshift Online is Red Hat's public Cloud application development and hosting platform that automates the provisioning, management and scaling of applications.[4] Openshift is the Cloud platform on which the Django framework is loaded which handles the complete processing of the system. Other modules loaded on Openshift are, namely- Python, MySQL and phpMyAdmin.

- Cloud Server: Django handles the interaction between the Cloud Client and the database. Django is developed with python. It uses an admin and a staff model for development of a Proposed System in its environment. It acts as a middleware in this system.
- Database: The complete database is stored in MySQL. PhpMyAdmin is used to configure and manipulate MySQL.

The initial setup required to develop this system is described in the following section.

\section{EXPERIMENTAL SETUP}

The experimental setup includes tools such as Git, Eclipse, Django, Python, on local computer. Eclipse is used to create .py format application for our Proposed System in Django Framework with Python 2.7 and test them on Cloud client. Git is a version control tool. It is used to upload and download application files created using Django on Cloud from Cloud Client The source files are PUSHED (uploaded) on Cloud and PULLED (downloaded) from the same drive. Git creates automatic log versioning of PUSH and PULL commands. The system is built with following configuration for the experimental setup as follows:

\subsection{Django}

Django is a high-level Python Web framework that encourages rapid development and clean, pragmatic design. It's free and open source. [5] The Proposed System application is built under Django framework with Python. It helps making the code easy by using built in libraries and features which bring benefit to the Proposed System. The Proposed System requires scalability, as users such as Patients and Doctors increases over time, Django provides best scalability features.

\subsection{Openshift}

Cloud based Health First is hosted on Openshift where all the python application of Cloud based function are stored. The application supports MySQL which enables it to store application data in MySQL format on Openshift host storage.

\subsection{Mysql}

MySQL is an open-source relational database management system. With Openshift it can be easily deploy and run applications backend by MySQL using Cloud servers and frameworks. Openshift Online currently supports MySQL 5.1 and 5.5. [6] The Proposed System comprises of storage of data such as medical records and login information. This data is stored using MySQL which is available in Openshift. The experimental setup requires safe and secure data with availability, also with Django it makes sure the data is secure and Cloud makes the data available all the time. We have concluded the core concept of the complete system in the following section.

\section{CONCLUSION}

This paper, presents a Cloud based Health First System which maintains user privacy, such that medical records can only be accessed by the authorized Doctors and the Patients. The information is maintained and stored by the proposed Health First system. This proposed model helps in organizing the records such as prescription, $\mathrm{x}$-rays and other types of reports in the cloud. This would eliminate the need to maintain records separately. Only the Doctor can upload and edit the records whereas Patients can only view his/her health or medical records. This paper also includes the architecture for the working model of the Cloud Based Health First System in detail.

Future Scope may include of features such as integrated Blood bank System. This can be used at the time of Emergency. 


\section{REFERENCES}

[1] J. Thomas, "Medical records and issues in negligence", Para. 1,Jul. 2009 [Online]. Available: http://www.ncbi.nlm.nih.gov/pmc/articles/PMC2779965/ [Accessed: Feb. 20, 2015].

[2] B. Ohlman, A. Eriksson, R. Rembarz, "What Networking of Information Can Do for Cloud Computing," , 18th IEEE International Workshops on Enabling Technologies: Infrastructures for Collaborative Enterprises

[3] Top Mobile Trends, "Mobile Tech Contributions to Healthcare \& Patient Experiences", Para. 5, 22 May 2014. [Online].

Available: http://topmobiletrends.com/mobile-technologycontributions-Patient-experience-parmar/ [Accessed: Feb. 20, 2015].

[4] Openshift, "OpenShift Online" Red Hat.Inc, para. 1, Available: https://www.openshift.com/products/online/ [Accessed: Feb. 20, 2015].

[5] Django, "Meet Django", para.1, Available: https://www.djangoproject.com/ [Accessed: Feb. 20, 2015].

[6] Openshift,"MySql on Openshift" Red Hat.Inc, para.1, Available:

https://developers.openshift.com/en/databasesmysql.html [Accessed: Feb. 20, 2015] 